\title{
Bimodal multispectral imaging system with cloud-based machine learning algorithm for real-time screening and detection of oral potentially malignant lesions and biopsy guidance
}

\author{
Narayanan Subhash $\odot$, ${ }^{\mathrm{a}, *}$ Suresh Anand $\odot,{ }^{\mathrm{a}}$ Ranimol Prasanna, \\ Sandeep P. Managoli, ${ }^{a}$ Rinoy Suvarnadas, ${ }^{a}$ Vidyarani Shyamsundar, \\ Karthika Nagarajan, ${ }^{\mathrm{b}}$ Sourav K. Mishra, ${ }^{\mathrm{c}}$ Migi Johnson, ${ }^{\mathrm{d}}$ \\ Mahesh Dathurao Ramanand, ${ }^{\mathrm{e}}$ Sanjay C. Jogigowda $\odot{ }^{\mathrm{f}}$ Vishal Rao, ${ }^{\mathrm{g}}$ \\ and Kodaganur S. Gopinath ${ }^{\mathrm{h}}$

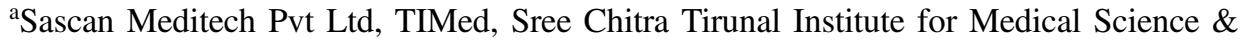 \\ Technology (SCTIMST), Thiruvananthapuram, Kerala, India \\ ${ }^{\mathrm{b}}$ Sree Balaji Dental College \& Hospital, Center for Oral Cancer Prevention Awareness \\ and Research, Chennai, Tamil Nadu, India \\ 'Institute of Medical Sciences and SUM Hospital, Department of Oncology, \\ Bhubaneswar, Orissa, India \\ ${ }^{\mathrm{d}}$ Government Dental College, Department of Oral Medicine and Radiology, \\ Kottayam, Kerala, India \\ ${ }^{\mathrm{e}}$ Dayananda Sagar College of Dental Sciences, Department of Oral Medicine, \\ Bangalore, Karnataka, India \\ ${ }^{\mathrm{f}}$ JSS Dental College \& Hospital, Department of Oral Medicine, Mysore, Karnataka, India \\ ${ }^{\mathrm{g}} \mathrm{HCG}$ Cancer Center, HCG Towers, Bengaluru, Karnataka, India \\ ${ }^{\text {h}}$ HCG Bangalore Institute of Oncology, Bengaluru, Karnataka, India
}

\begin{abstract}
Significance: Screening and early detection of oral potentially malignant lesions (OPMLs) are of great significance in reducing the mortality rates associated with head and neck malignancies. Intra-oral multispectral optical imaging of tissues in conjunction with cloud-based machine learning (CBML) can be used to detect oral precancers at the point-of-care (POC) and guide the clinician to the most malignant site for biopsy.

Aim: Develop a bimodal multispectral imaging system (BMIS) combining tissue autofluorescence and diffuse reflectance (DR) for mapping changes in oxygenated hemoglobin $\left(\mathrm{HbO}_{2}\right)$ absorption in the oral mucosa, quantifying tissue abnormalities, and guiding biopsies.

Approach: The hand-held widefield BMIS consisting of LEDs emitting at 405, 545, 575, and $610 \mathrm{~nm}, 5 \mathrm{MPx}$ monochrome camera, and proprietary Windows-based software was developed for image capture, processing, and analytics. The DR image ratio (R610/R545) was compared with pathologic classification to develop a CBML algorithm for real-time assessment of tissue status at the POC.

Results: Sensitivity of $97.5 \%$ and specificity of $92.5 \%$ were achieved for discrimination of OPML from patient normal in 40 sites, whereas $82 \%$ sensitivity and $96.6 \%$ specificity were obtained for discrimination of abnormal (OPML + SCC) in 89 sites. Site-specific algorithms derived for buccal mucosa (27 sites) showed improved sensitivity and specificity of $96.3 \%$ for discrimination of OPML from normal.

Conclusions: Assessment of oral cancer risk is possible by mapping of $\mathrm{HbO}_{2}$ absorption in tissues, and the BMIS system developed appears to be suitable for biopsy guidance and early detection of oral cancers.
\end{abstract}

*Address all correspondence to Narayanan Subhash, subhash.sascan@gmail.com 
(C) The Authors. Published by SPIE under a Creative Commons Attribution 4.0 Unported License. Distribution or reproduction of this work in whole or in part requires full attribution of the original publication, including its DOI. [DOI: 10.1117/1.JBO.26.8.086003]

Keywords: wide-field diffuse reflectance imaging; multispectral bimodal imaging; oral potentially malignant lesions; oxygenated hemoglobin absorption mapping; biopsy guidance; oral cancer screening and early detection.

Paper 210148R received May 6, 2021; accepted for publication Jul. 26, 2021; published online Aug. 16, 2021.

\section{Introduction}

According to GLOBOSCAN 2018 reports, 354,864 new cases of oral cancer and 177,384 deaths have occurred worldwide in $2018,{ }^{1}$ and a fifth of this burden is from India. ${ }^{2}$ The five-year survival rate for oral cancers is around $50 \%$, regardless of the improvement in diagnostic modalities and treatment outcomes. ${ }^{3}$ Early detection of pre-malignant lesions in the oral cavity is the best way to improve the quality of life of patients, to effectively manage the disease, and to improve treatment outcomes. ${ }^{4}$ In oral cancer diagnostics, detection of oral potentially malignant lesions (OPMLs) is of great significance. OPML such as leukoplakia, erythroplakia, and oral submucous fibrosis (OSMF) have a risk for malignant transformation of $15 \%$ to $39 \%, 51 \%$, and $7 \%$ to $26 \%$, respectively. ${ }^{5}$ Recent guidelines recommend an initial biopsy to assess the disease status of OPMLs. ${ }^{6}$ Tissue biopsy is known to be the diagnostic gold standard for OPML identification and analysis. ${ }^{7,8}$

In low-resource settings, conventional oral examination (COE) with white light is the standard procedure for evaluating OPMLs. ${ }^{6}$ The major limitation associated with COE is that it is subjective; the screening results depend on the expertise of the clinician examining the patient. Often, malignant tissue changes are discarded by $\mathrm{COE},{ }^{9}$ and dysplastic tissues can still be located within healthy oral mucosa. ${ }^{10}$ Evidence shows that COE is not a good discriminator of oral mucosal lesions. ${ }^{11}$ Clinical examination has limited value in detecting the malignant potential of OPMLs since their macroscopic appearance often does not reflect their histopathologic and molecular features; despite this, evaluation of OPMLs is still largely based on simple mucosal inspection. ${ }^{12}$ It is a challenging task even for experienced clinicians to locate the most malignant site for biopsy, especially in large OPMLs. This anomaly often leads to multiple or unwanted biopsies, delayed- and under-diagnosis, and patient trauma. ${ }^{13}$ Because tissue biopsies are intrusive, labor-intensive, and often take a few days to process and interpret, many OPMLs are not biopsied, particularly in low-resource settings. ${ }^{14}$ The existing adjunctive techniques, including vital tissue staining, brush biopsy, chemiluminescence, and autofluorescence imaging, do not provide sufficient diagnostic accuracy for the detection of premalignant changes in the oral mucosa. $^{13,15}$

Tissue progression from healthy to malignant states is followed by several biochemical, morphological, and structural changes associated with the disease. ${ }^{16}$ These changes mirror the optical signatures derived from the interaction of light with tissues. Autofluorescence emanating from coenzymes, such as nicotinamide adenine dinucleotide (NAD), flavin adenine dinucleotide (FAD), and protoporphyrin IX (PpIX), present information on cellular metabolic activity. In contrast, reflectance spectroscopy can be used as biomarkers of changes in the tissue oxygenation levels and neovascularization. ${ }^{17}$ A screening device should ideally have the ability to detect early tissue transformations toward malignancy, enabling better patient care and improved survival rates.

Several clinical studies have been performed by our group ${ }^{18-21}$ and other researchers ${ }^{22-26}$ on the application of optical techniques for the detection of oral premalignant lesions. These studies have shown that non-invasive clinical adjuncts based on tissue fluorescence and diffuse reflectance spectroscopy have great potential as screening tools for the detection of oral malignancies. Our approach utilized the changes in the intensity of oxygenated hemoglobin absorption peaks at 545 and $575 \mathrm{~nm}$, which were noticed in the diffusely reflected white light for classifying oral malignancies. Initial studies were carried out ex vivo, on surgically excised tissues of oral 
cavity, ${ }^{18}$ and were later validated through an in vivo clinical study.$^{19}$ We recorded the diffusely reflected white light spectra from the oral mucosa with a fiber-optic probe and noticed that the diffuse reflectance (DR) intensity ratio (R545/R575) was lowest in normal or healthy mucosa and gradually increased to higher and higher values with increasing grades of oral cancer. The heme production is low in cancer cells owing to the reduced activity of ferrochelatase enzyme in the heme cycle, which leads to a build-up of PpIX in the cancer cells. ${ }^{19}$ By recording the $405-\mathrm{nm}$ laser-induced fluorescence spectra of oral mucosa with an optical fiber probe, we were able to detect this increase in PpIX in vivo and discriminate different grades of oral cancer using a spectral ratio reference standard. ${ }^{20}$ Later, a widefield imaging configuration was developed with white light illumination and an external electron multiplying CCD camera to capture DR images of oral lesions at 545 and $575 \mathrm{~nm}$, and the image ratio R545/R575 was utilized to screen and detect oral cancers. ${ }^{21}$

Most of the commercially available devices in the market, such as VELscope ${ }^{\circledR}$ (LED Dental, White Rock, British Columbia, Canada) (20 to 22), Bio/Screen ${ }^{\circledR}$ (Addent, CT), ViziLite PRO ${ }^{\circledR}$ (DenMat, Lompoc, California), and OralID ${ }^{\circledR}$ (Forward Science), rely on visualization of tissue fluorescence on excitation with violet/blue light from outside of the oral cavity, whereas Identafi $^{\circledR}$ (StarDental - DentalEZ, Lancaster, Pennsylvania) is an intraoral device that uses both tissue fluorescence and reflectance of tissues for oral cancer screening. ${ }^{27-29}$ However, the main limitation with these devices is that they are subjective, relying mostly on visual impressions; however, VELscope, Bio/Screen, and Vizilite $\mathrm{PRO}^{\circledR}$ do have options for external camera attachment. ${ }^{30}$ Cancerous lesions often show loss of fluorescence and appear as dark areas in these images, making it impractical for a biopsy guidance application. The detection accuracies reported by these devices are poor as inflammatory tissues also show loss of fluorescence, and unwanted biopsies are a major concern. Therefore, these devices did not gain acceptance even in countries such as India where the prevalence for oral cancer is high. Although, Identafi ${ }^{\circledR}$ is an intraoral bimodal device, it also is subjective and does not incorporate a camera for image capture. ${ }^{31,32}$ Furthermore, the use of an expensive disposable mirror adds to the cost of screening and makes it unsuited for population-based screening programs.

These limitations motivated us to develop point-of-care (POC) solutions that provide quantitative information on tissue status at the POC in real-time. In this paper, we present a hand-held multispectral wide-field imaging intraoral camera for recording of tissue autofluorescence and DR that is illumined with multiple LEDs. The monochrome USB camera is controlled through proprietary software installed on a personal computer. The captured images are processed and analyzed using a cloud-based machine-learning (ML) algorithm for real-time user feedback. With the help of pseudo-color maps representing variations in oxygenated hemoglobin absorption in tissue, we detect tissue abnormalities and locate the most malignant site in a lesion for biopsy. We also present a representative pilot study results to demonstrate the potential of the device for screening and early detection of oral cancers and guided biopsies.

\section{Materials and Methods}

\subsection{Instrumentation}

A hand-held bimodal multispectral imaging system (BMIS) has been developed [Figs. 1(a) and 1(b)] for wide-field intra-oral screening. ${ }^{33}$ The BMIS consists of a sub-miniature monochrome camera (Ximea, GmbH, Model: MU9PM-MH) featuring a 5 MPx CMOS sensor (Aptina MT9P031) with 2.2-micron pixels and $2592 \times 1944$ resolution for image capture (Fig. 1). Light-emitting diodes (LEDs) situated around the camera lens, emitting at violet $(405 \mathrm{~nm})$, green $(545 \mathrm{~nm})$, yellow $(575 \mathrm{~nm})$, and red $(610 \mathrm{~nm})$ wavelengths of light, were used for tissue illumination. Narrowband interference filters (5-mm diameter, FWHM width of $8 \mathrm{~nm}$ ) centered around 542,577 , and $610 \mathrm{~nm}$ were placed over a window covering the respective LEDs to ensure that their light output matches with the absorption dips of $\mathrm{HbO}_{2}$ at 542 and $577 \mathrm{~nm}$ and that the LEDs spectral outputs do not overlap. The 610-nm LED acts as a reference standard, in which $\mathrm{HbO}_{2}$ absorption is minimal. The light collection optics included a tailored filter that transmits tissue autofluorescence and the elastically backscattered light at 542 and $577 \mathrm{~nm}$ into the sensor, while 


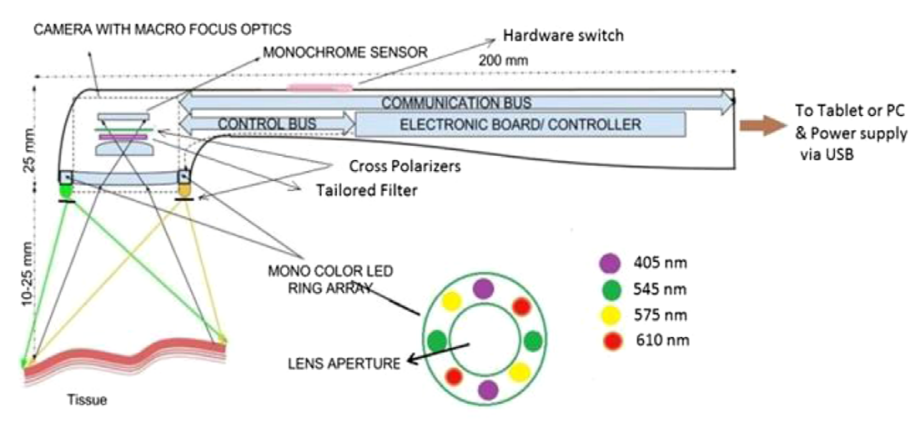

(a)

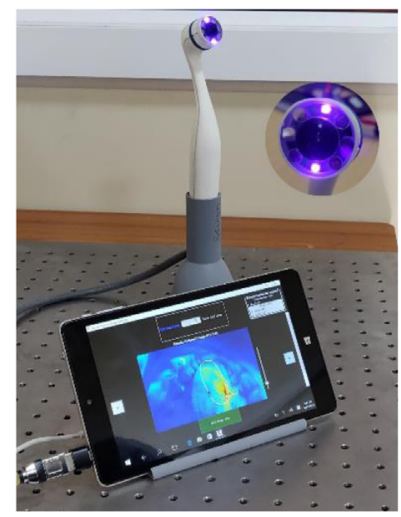

(b)

Fig. 1 (a) Schematic of the BIMS system developed for oral cancer screening and (b) prototype of the BMIS connected to an 8 in. Windows tablet. The inset shows the probe head with narrowband LED filters mounted on the outer window.

blocking the 405-nm light from entering the detection system. The patented optical engine also consisted of crossed polarizers in the light illumination and collection paths to prevent specular reflections from the tissue surface reaching the sensor. The camera is connected to the USB port of a tablet or laptop with a 64-bit Windows 10 operating system [Fig. 1(b)]. The BMIS is thus configured to capture multimodal images of oral mucosa using its integrated hardware and proprietary software. More details on the BIMS imaging system regarding optical configuration, hardware, and software integration are described elsewhere. ${ }^{33}$

\subsection{Tissue Classification Algorithm}

In the present study, we implemented a ratio-metric algorithm (R545/R610) based on the diffusely reflected light intensities of 545 and $610 \mathrm{~nm}$. This was achieved by pixel-by-pixel division of the monochrome images captured by the camera following illumination with 545- and 610-nm wavelength LEDs. The DR intensity of tissues $(R)$ is determined by the concentration of $\mathrm{HbO}_{2}$ and $\mathrm{Hb}$ in tissues and their effective attenuation coefficients ( $a 1$ and $a 2)$ at these wavelengths using the relation: ${ }^{34}$

$\ln (R)=\left(a 1 *\left[\mathrm{HbO}_{2}\right]+a 2 *[\mathrm{Hb}]\right)+\ln (k)$, where $k$ is a constant term, which can be removed by calibration, and total hemoglobin concentration $(\mathrm{tHb})$ and oxygen saturation $\left(\mathrm{S}_{\mathrm{t}} \mathrm{O}_{2}\right)$ are defined as:

$$
\mathrm{tHb}=\left[\mathrm{HbO}_{2}\right]+[\mathrm{Hb}], \quad \text { and } \quad \mathrm{S}_{\mathrm{t}} \mathrm{O}_{2}=\left[\mathrm{HbO}_{2}\right] / \mathrm{t}[\mathrm{Hb}] .
$$

The DR intensity at any two wavelengths can be used to calculate the concentration of $\mathrm{HbO}_{2}$ and $\mathrm{Hb}$ so that the total concentration of hemoglobin and oxygenation can be determined. The wavelength $545 \mathrm{~nm}$ belongs to one of the isosbestic points of $\mathrm{Hb}$, where $\mathrm{HbO}_{2}$ and $\mathrm{Hb}$ have strong absorption. At this wavelength, the reflectance intensity is more sensitive to changes in $\mathrm{HbO}_{2}$ than at other wavelengths in the spectrum. In the 610- to 630-nm wavelength range, the absorption coefficient of $\mathrm{Hb}$ is 7 to 8 times higher than that of $\mathrm{HbO}_{2}$ so that absorption at this wavelength is primarily due to $\mathrm{Hb}$ present in the tissue (Fig. S1 in the Supplementary Material). Therefore, the extent of malignant transformation can be assessed from the increase in the R610/R545 ratio in the region of interest (ROI) marked surrounding the lesion, with reference to the tissue fluorescence image (F405).

It is known that reflectance and light scattering spectroscopy provide information on morphologic and structural changes in tissue architecture and epithelial cell nuclei and polarized light reflectance spectroscopy provides quantitative information on tissue morphology that could be used for non-invasive and real-time detection of epithelial neoplasia. ${ }^{35}$ The tissues of various anatomical sites of the oral cavity have differing optical and spectral characteristics. Therefore, we grouped the tissues of different anatomical sites into keratinized and non-keratinized types, 
with the former consisting of gingiva, vermillion border of the lip, dorsal tongue, hard palette, and lower/upper alveolus and the latter consisting of left/right buccal mucosa, lateral/ventral tongue, floor of the mouth, inner lip, and gingivo-lingual/buccal sulcus. Therefore, two sets of R610/R545 ratio algorithms were developed to provide user feedback during the screening process, based on the site of the lesion. When biopsies are taken from the sites identified as most malignant, the R610/R545 value associated with the biopsy site is uploaded to the cloud along with the pathology report. The new ratio values and the corresponding diagnostic results are merged into the ML algorithm, making it robust over a period of time. When more and more data sets are incorporated into the algorithm and site-specific algorithms become available, the accuracy of screening and disease prediction improves.

\subsection{Clinical Trials}

The BMIS was validated through a multicentric clinical study covering six hospitals (HCG Hospital, JSS Dental College, SUM Hospital, Govt Dental College Kottayam, Sri Balaji Dental Hospital, and Dayanand Sagar Dental College). Individual ethical approvals were obtained from the respective ethics committees of these hospitals, and the trial was registered prospectively at the Clinical Trial Registry of India with Ref No. CTRI/2017/10/010125 dated October 18,2017 . The study details were explained in detail to the patients who participated in the study, and written informed consent was obtained before initiation of any study-related procedures.

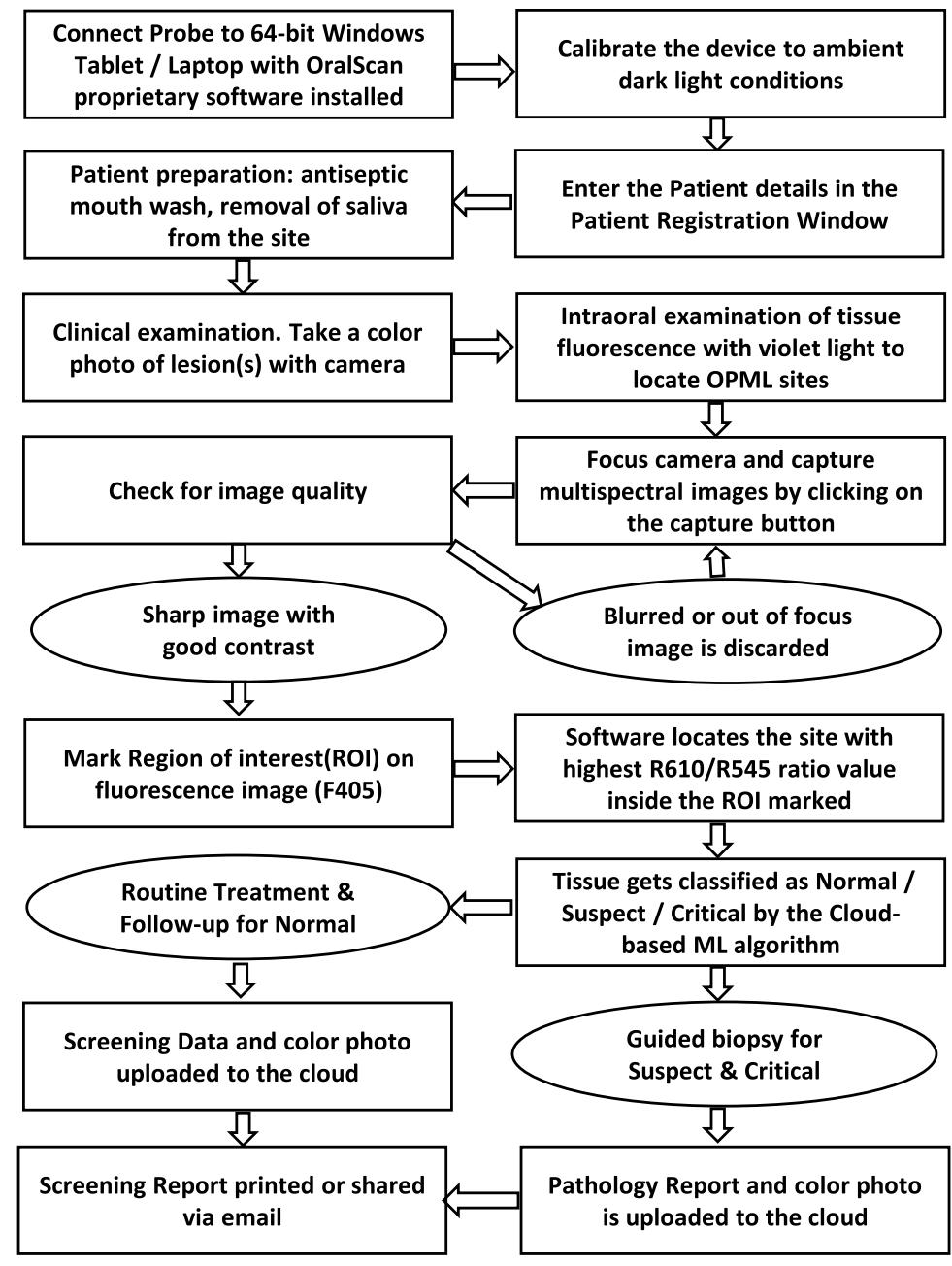

Fig. 2 Schematic diagram showing the working process of the BMIS. 
All clinical measurements using the BIMS probe were carried out in a dark room following the calibration procedure given in Sec. 2.5. Figure 2 shows a schematic diagram of the steps involved in the working process of the instrument. The entire screening process takes no more than 5 mins to complete for a patient. The immediate display of the captured images on the computer screen helps with image quality checks and recapture when necessary. Once the ROIs are marked with reference to the tissue autofluorescence image, the software program locates the pixel with the maximum ratio value in the ROI and assigns the mean value of a $5 \times 5$ matrix surrounding that pixel as the DR ratio (R610/R545) value for tissue classification as normal, suspect, or critical based on feedback from the cloud-based ML algorithm. A guided biopsy shall be taken if required. The graphical user interface (GUI) and work flow are organized to make the screening process intuitive and straightforward. The screening data is saved in the local drive of the computer and uploaded to the cloud for backup and algorithm refinement. The storage and integration of patient data from different screening centers on a single cloud platform facilitate uniformity in analytics and development of a robust ML algorithm for the benefit of all users.

\subsection{General Description of the Data Set}

A total of 336 patients were recruited for this study, out of which 89 patients (118 sites) underwent biopsy procedures. Multiple sites of the same patient showing different grades of pathology were included as a separate dataset. Images from 118 intraoral sites were further scrutinized; four data were excluded due to lack of histopathologic report, six data were removed due to poor image quality (blurring), and 10 data were excluded due to non-uniformity in light illumination. Finally, 89 sites from 65 patients were included in the study. The average age $( \pm S D)$ of the patients was $51 \pm(14)$ years. The inclusion criteria incorporated selection of patients presenting with OPML, such as leukoplakia, erythroplakia, OSMF, dysplasia, and moderate to well-differentiated squamous cell carcinoma (SCC) in the case of malignant tissues. The exclusion criteria encompassed patients who have undergone prior cancer treatments, have systemic conditions that contraindicate biopsy, have used any oral medication for at least four weeks, or were not willing to participate in the study. OSMF cases are mostly diagnosed from their clinical characteristics; hence they are not biopsied as a surgical intervention may induce further disease progression. Various types of pathological specimens screened using the probe are given in Table 1.

Table 1 Clinical status of different pathological specimens included in the study.

\begin{tabular}{lc}
\hline \hline Tissue type & \# of patients \\
\hline OPML & 1 \\
Carcinoma in situ & 2 \\
Lichen planus & 1 \\
Fibro epithelial polyp & 27 \\
Leukoplakia & 8 \\
Hyperplasia & 1 \\
Ulcer & \\
Malignant lesions & 7 \\
Poorly differentiated SCC & 14 \\
Moderately differentiated SCC & 28 \\
Well differentiated SCC
\end{tabular}




\subsection{Calibration of the Instrument}

The BMIS is calibrated to the ambient (dark/dim) light conditions by positioning the probe head over the calibration unit that houses a tissue phantom, maintaining a working distance of $2 \mathrm{~cm}$ with the probe tip. The calibration process is initiated by clicking on the calibration/settings button on the software window. The display shows calibration successful on completion of the procedure that involves switching on/off of the different LEDs and varying the exposure settings of the camera for each set of LEDs such that all four images captured by the camera are optimally exposed during the recording process. This is achieved by limiting the mean pixel intensity of the four different frames to within $2.5 \%$ to $4 \%$ of their mean pixel value based on the number of retries done to successfully calibrate the device. Once calibrated, there is no need to recalibrate the instrument unless the room lighting conditions change.

\subsection{Image Acquisition and Analysis of the Tissue Characteristics}

During patient enrollment, in addition to patient name and contact details, mandatory information related to age, habits, and visual/clinical impressions are collected, whereas the medical record number is autogenerated [Fig. 3(a)] in the patient information section of the software program. The patients are seated comfortably on a dental chair and are examined following the sequence shown in Fig. 2. An oral rinse with water followed by saline wash or antibacterial mouthwash is recommended. The lesion photo is captured on a mobile phone camera for reference. The screening probe is now wiped cleaned with isopropyl alcohol and covered with a thin and transparent plastic cling wrap film (Polyvinyl Films, Inc, Massachusetts, Model: Kirkland stretch-tite or equivalent food wrap film) to maintain hygiene and prevent probe contact with the oral cavity. The room light is set to the ambient dark conditions used for calibration. On powering the probe, the violet LED is switched on. This light is used to observe tissue abnormalities, locate OPMLs by tissue autofluorescence, and identify areas for detailed examination.

The software has provisions for marking the suspicious sites for detailed investigation in an anatomical diagram based on visual observation. On selecting the imaging mode, a live view of the oral cavity is possible in a new window [Fig. 3(d)] in monochrome (grayscale) or pseudo color to locate the lesion spread and visually assess the extent of tissue health. The software has provisions for video and image capture. During screening, care should be taken to minimize specular reflection from oral mucosa by keeping the mucosal surface dry with cotton swab and holding the probe at an angle to the imaging surface. Once the OPML is located, the camera is focused by varying the distance between the probe and the tissue, and four monochrome images are captured sequentially by a single press of the capture button on the device or the software window. The quality of the images displayed on the screen [Fig. 3(e)] is checked to ensure that all four images are sharp without motion artifacts due to patient or probe movement and are free of any bright spots due to reflection from saliva, explorer probe mirror, or illumination nonuniformity. Although the image capture takes $<90 \mathrm{~s}$, probe movement can be minimized by resting the hand holding the probe on the patient's body or some other stationary object. If the images are not focused or intensity saturation is noticed in any region, the captured images can be discarded and a fresh set of images recaptured.

The four images captured sequentially and illuminated with four different LEDs emitting at 405, 545, 575, and $610 \mathrm{~nm}$ are designed at F405, R545, R575, and R610. All four monochrome images are displayed on screen along with the R610/R545, R610/R575, and R545/R575 ratio images in monochrome/pseudo color [Fig. 3(e)]. The outer border of the OPML or malignant lesion is marked as the ROI using the mouse pointer or stylus pen on the touch screen based on the extent of the pseudo color map (PCM) of tissue fluorescence. In addition, an apparently healthy region that is free of tissue inflammation is marked on the same anatomical region adjoining the lesion with a low R610/R575 ratio value ${ }^{36}$ [Fig. 3(f)]. The software program automatically determines the mean DR image ratio (R610/R545 and R610/R575) in the ROIs of healthy mucosa and the highest ratio value in the ROIs of R610/R545, R545/R575, and R610/R575 in the OPML. The DR ratio values along with the processed and captured images are now pseudo color mapped to visualize variations in the DR ratio across the lesion. 


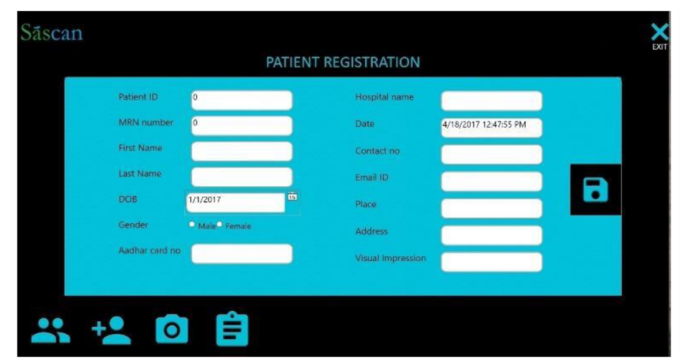

(a)

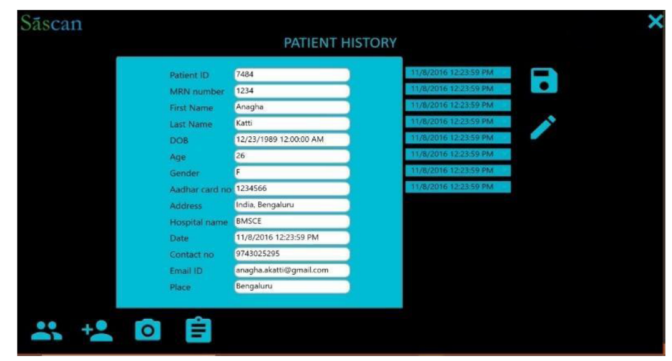

(c)

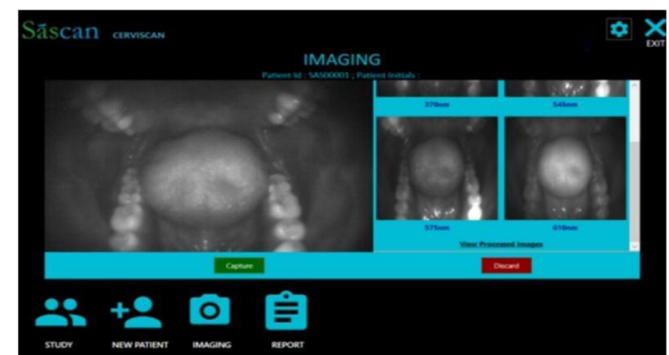

(e)

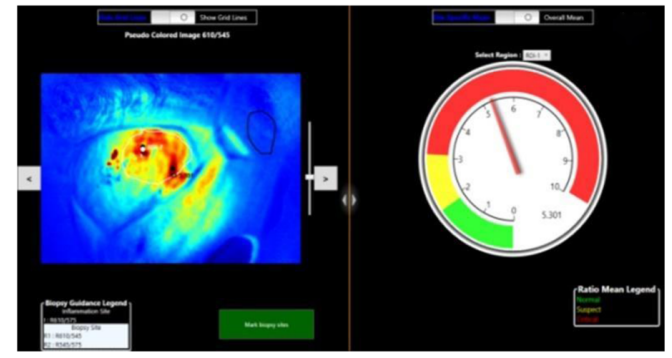

(g)

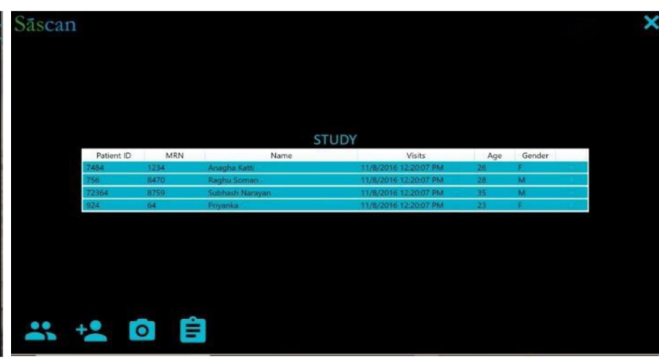

(b)

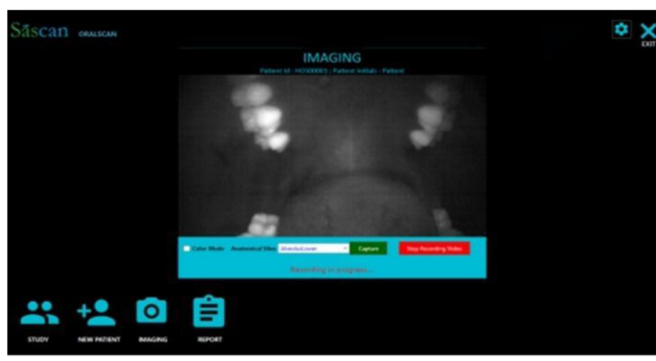

(d)

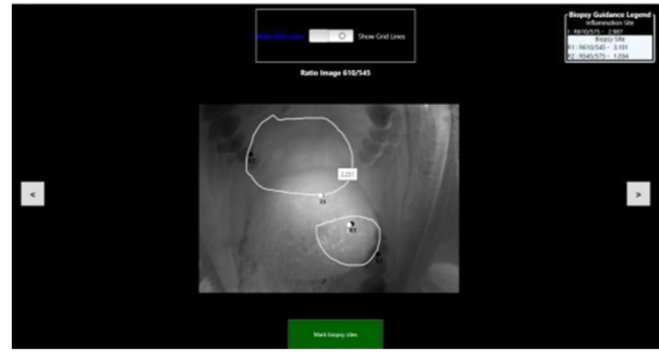

(f)

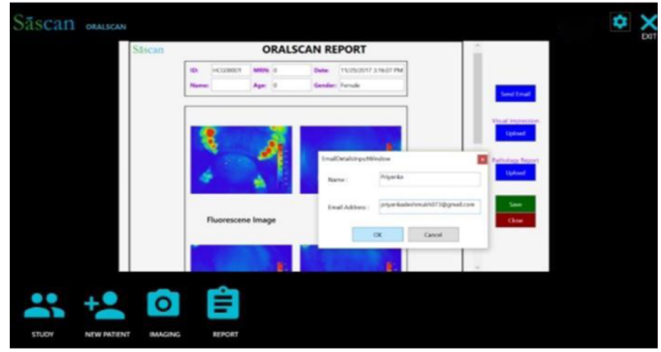

(h)

Fig. 3 Different modules of BMIS software for image capture and analysis. (a) Opening window for patient registration; (b) patient study window to select patient for screening; (c) patient history window for viewing/editing of captured images; (d) video recording window; (e) image capture and display window; (f) window for ROI marking of patient normal and abnormal areas; (g) PCM of R610/R545 image ratio for biopsy guidance, and the CDD for tissue status assessment, with green representing normal, yellow representing OPML (Suspect), and red representing SCC (Critical); and (h) report page with features for biopsy result entry, data upload to the cloud server, and report sharing.

The extent of color coding can be altered by up or down movements of the cursor on the PCM adjuster located beside the image display window. The screening result is presented in a colorcoded display diagram (CDD), with the pointer showing the highest R610/R545 ratio value in the ROI, representing the most malignant site in the OPML. Green color in the CDD represents healthy/normal tissue, yellow represents suspect (OPML), and red represents critical (malignant) lesions. 
Based on the variance of the R610/R545 ratio value representing the most malignant site in the ROI with respect to the mean ratio value of an adjoining healthy region, it would be possible for the clinician to decide on whether a biopsy is required or habit cessation and follow-up would suffice. On completion of the screening process, the value of DR ratios, such as R610/R545, R545/R575, and R610/R575, are populated in an excel file, and the captured images and data files are pushed to the cloud automatically on internet connectivity. Later, when the ROI is remapped or the pathology reports and lesion photos are uploaded, the cloud data also are refreshed automatically.

\section{Results}

Fluorescence visualization of homogenous leukoplakia in the right buccal mucosa of a patient with violet light illumination is depicted along with the PCM of the corresponding autofluorescence and the DR image ratio (R610/R545) in Figs. 4(a)-4(d). The PCM of tissue fluorescence and DR ratio maps can be visually enhanced as per user perspective to locate the lesion spread. Figures 5(a)-5(d) show the PCM images tissue fluorescence, the DR image ratio R610/R545 representing malignant transformations, R610/R575 representing tissue inflammation, and the photo of the oral cavity of the patient with speckled leukoplakia on the left buccal mucosa. The ROI of the lesion is marked on the fluorescence image outlining the lesion, while the ROI representing patient normal is marked in an adjoining area belonging to the same anatomical site, carefully avoiding areas of tissue inflammation with high R610/R575 values. Once the ROI is marked, the user can visualize the most malignant site in the lesion for biopsy from the PCM of the R610/R545 ratio that represents the absorption changes due to $\mathrm{HbO}_{2}$ in tissue. The corresponding ratio value with respect to the most malignant site also is displayed on the screen along with the mean value of the DR image ratio from the adjoining site marked as patient healthy. As the mouse pointer has a ratio drop-down display feature, the DR ratio values across the lesion can be explored by moving the mouse cursor across the lesion to review and locate if any malignant sites of the oral cavity went unnoticed during the ROI marking process.

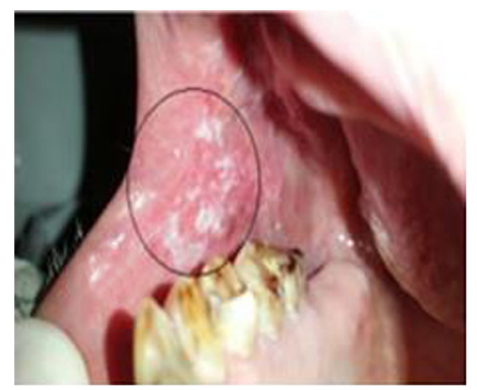

(a)

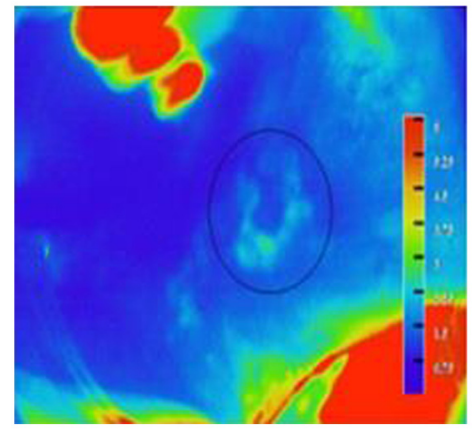

(c)

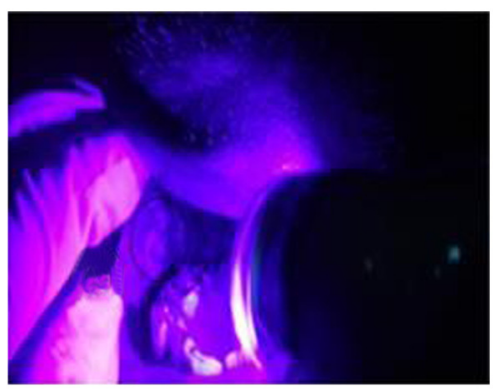

(b)

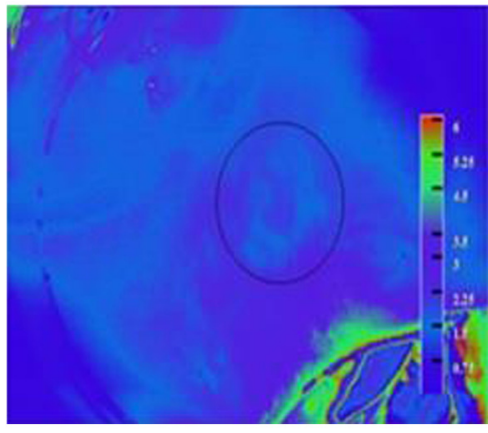

(d)

Fig. 4 Homogenous leukoplakia on the right buccal mucosa: (a) photo of the lesion, (b) live view with violet light, (c) PCM of tissue fluorescence, and (d) PCM of R610/R545 image ratio. 


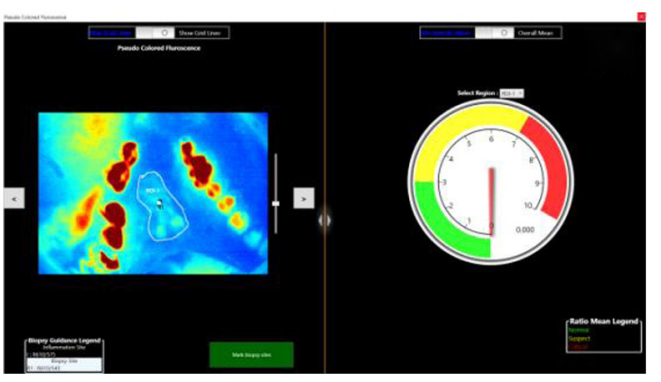

(a)

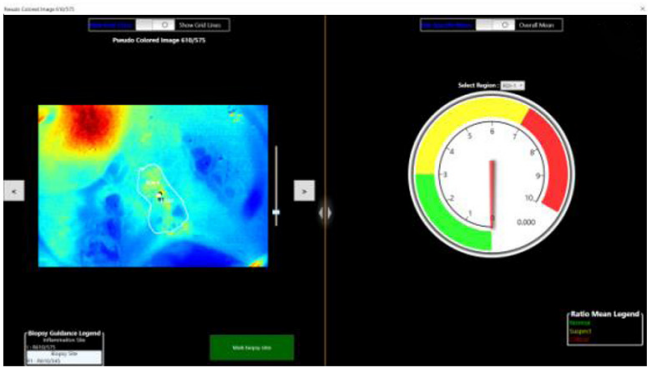

(c)

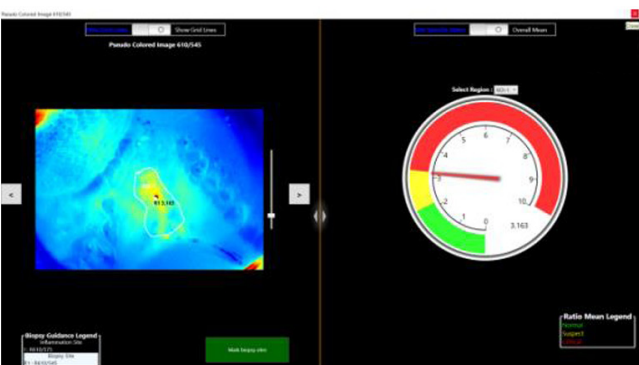

(b)

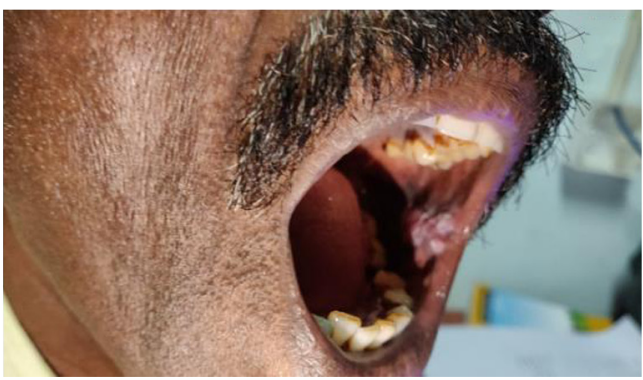

(d)

Fig. 5 Screening results of a 59-year-old patient with speckled leukoplakia on the left buccal mucosa: (a) PCM of tissue fluorescence, (b) PCM of R610/R545 image ratio with the tissue status displayed on the CDD, (c) image ration R610/R575 representing tissue inflammation, and (d) lesion photo. The lesion border is marked on the screen based on visual impression and PCM of tissue fluorescence. The software program locates the site with the highest ratio value, inside the white ROI marked on the PCM of R610/R545 ratio, as the most malignant site for biopsy.

\subsection{Machine Learning Algorithm Development}

To classify the various tissue types involved in the study, we utilized the ratio (R610/R545) of the diffusely reflected light from squamous epithelium at $610 \mathrm{~nm}$ (R610) and $545 \mathrm{~nm}$ (R545). Figure 6 shows scatter plot diagrams representing the DR image ratio (R610/R545) versus patient number for discriminating potentially malignant (OPMLs) and abnormal tissues involving OPMLs and SCC, from apparently healthy tissues in patients. The tissues included in the algorithm were of varied morphology and structure, covering all anatomical locations in the oral

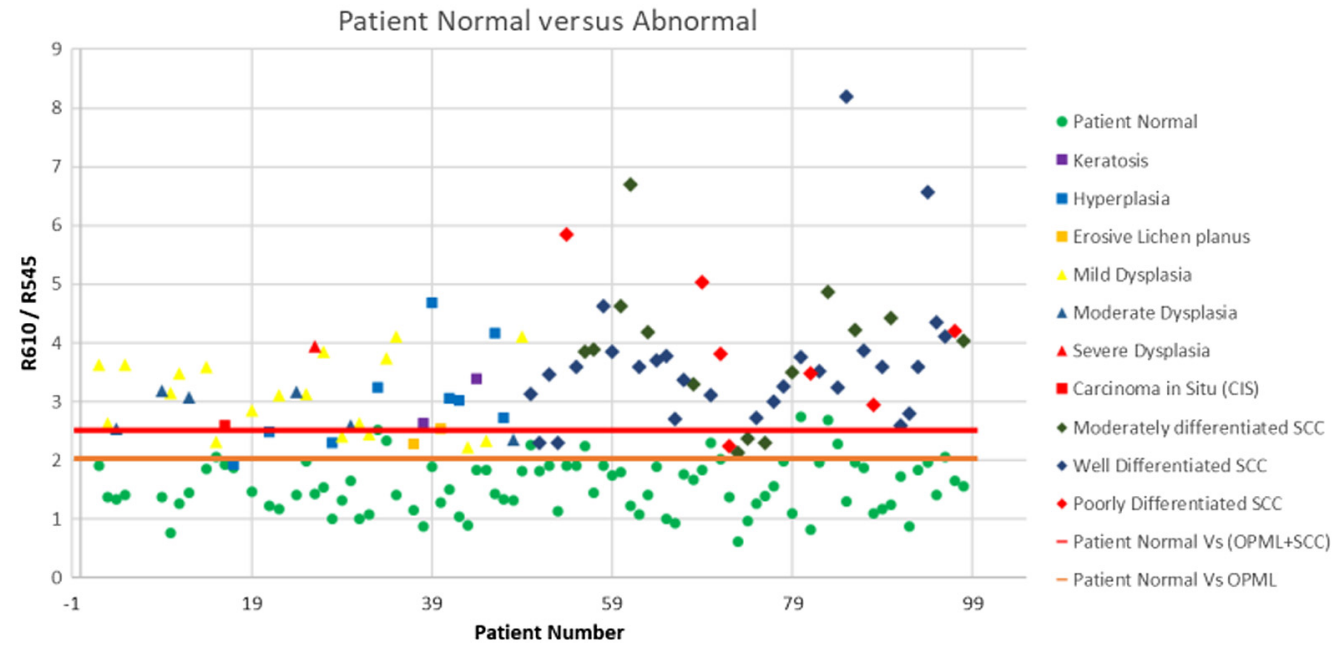

Fig. 6 Scatter plot ratio (R545/R610) algorithm for classifying abnormal and OPML tissues of the oral cavity from patient normal tissues, with the red discrimination line drawn at the mean of (OMPL + SCC) ratio values and the patient normal ratio values and the orange line drawn at the mean of OPML and patient normal ratio values. 
cavity (Table S1 in the Supplementary Material). The DR ratio values of healthy tissues relate to the mean R610/R545 ratio of the adjoining healthy region marked in the patient mucosa belonging to the same anatomical site, with no or minimal signs of tissue inflammation. ${ }^{36}$

The mean DR intensity ratio (R610/R545) for healthy samples was found to be 1.56 , whereas for OPML sites it was 3.03 and for SCC tissues the value was higher at 3.44. This shows that the DR image intensity ratio (R610/R545) increases with the increase in malignancy, with the lowest values for normal/healthy mucosa and increasing to higher and higher values as the tissue transformation takes place from OPML to SCC. The discrimination threshold lines shown in Fig. 6 were drawn at the mean values of the data sets involved. For instance, the discrimination threshold line between healthy tissues and OPML was calculated and drawn at the mean of the average ratio values of patient healthy and OPML data sets. In Fig. 6, the discrimination threshold lines were drawn at 2.028 and 2.501, respectively, for classifying OPML and abnormal (OPML+SCC) tissues from patient normal tissues covering all anatomical sites of patients that underwent a guided biopsy procedure.

\subsection{Diagnostic Accuracy}

Table 2 represents the diagnostic ability of the device obtained with the DR ratio (R610/R545) algorithm for discriminating OPML and abnormal tissues (OPML + SCC) from all anatomical sites with adjoining normal tissues from the same anatomical site. Table 2 also presents the improvement in diagnostic accuracy when healthy volunteer data are used instead of patient normal tissues in the R610/R545 ratio algorithm for buccal mucosal malignancies. To evaluate the diagnostic accuracy, we implemented the discrimination threshold as a cutoff value for differentiating healthy tissues from the different tissue pathologies involved. The algorithm shown in Fig. 6 for discriminating OPML from patient normal tissues with a cut-off value at 2.03 resulted in a sensitivity of $97.5 \%$ and specificity of $92.5 \%$ with positive predictive value (PPV) and negative predictive value (NPV) values of 0.93 and 0.97 , respectively, whereas for discrimination of patient normal tissues from abnormal tissues, a sensitivity of $82 \%$ and specificity of $96.6 \%$ were obtained with the cutoff value at 2.5 and PPV and NPV values of 0.96 and 0.84 , respectively.

The receiver operator characteristic-area under the curve (ROC-AUC) was also computed to evaluate the diagnostic performance of the R610/R545 ratio algorithm for tissue discrimination. The ROC-AUC values (Fig. 7) were found to be 0.987 and 0.989 , respectively, for discrimination of OPML tissues and abnormal (OPML + SCC) tissues from patient normal tissues. The

Table 2 Diagnostic ability of R610/R545 ratio for differentiating patient normal tissues from OPML lesions and abnormal tissues (OPML and SCC).

\begin{tabular}{|c|c|c|c|c|c|}
\hline \multirow[b]{2}{*}{$\begin{array}{l}\text { Diagnostic } \\
\text { parameters }\end{array}$} & \multicolumn{2}{|c|}{ All anatomical sites } & \multicolumn{3}{|c|}{ Buccal mucosa } \\
\hline & $\begin{array}{c}\text { Patient } \\
\text { normal } \\
\text { versus OPML }\end{array}$ & $\begin{array}{l}\text { Patient normal } \\
\text { versus abnormal } \\
\text { (OPML + SCC) }\end{array}$ & $\begin{array}{c}\text { Patient } \\
\text { normal } \\
\text { versus OPML }\end{array}$ & $\begin{array}{c}\text { Healthy } \\
\text { volunteer } \\
\text { versus OPML }\end{array}$ & $\begin{array}{c}\text { Healthy } \\
\text { volunteer versus } \\
\text { abnormal }\end{array}$ \\
\hline Sample size (n) & 40 & 89 & 27 & 27 & 43 \\
\hline Cut-off value & 2.028 & 2.501 & 2.214 & 2.112 & 2.287 \\
\hline Sensitivity (\%) & 97.5 & 82.02 & 96.3 & 96.3 & 95.34 \\
\hline Specificity (\%) & 92.5 & 96.63 & 96.3 & 100 & 100 \\
\hline $\begin{array}{l}\text { Positive predictive } \\
\text { value (PPV) }\end{array}$ & 0.929 & 0.961 & 0.963 & 1.00 & 1.00 \\
\hline $\begin{array}{l}\text { Negative predictive } \\
\text { value (NPV) }\end{array}$ & 0.974 & 0.843 & 0.963 & 0.964 & 0.955 \\
\hline ROC-AUC & 0.987 & 0.989 & 0.995 & 1.00 & 1.00 \\
\hline
\end{tabular}


Patient Normal versus OPML

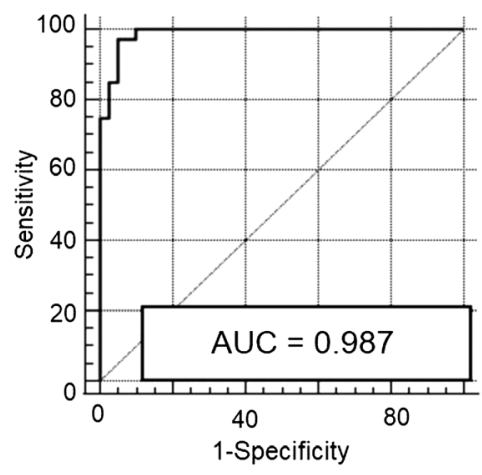

(a)

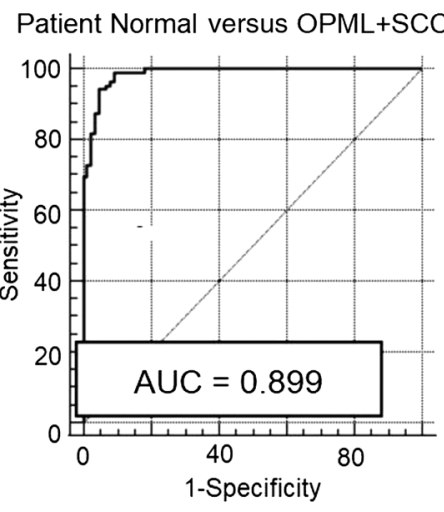

(b)

Fig. 7 ROC-AUC using R610/R545 intensity ratios for classifying (a) patient normal versus OPML sites and (b) patient normal versus abnormal (OPML + SCC) tissues in the oral cavity.

diagnostic accuracies given in Table 2 establish the significance of utilizing a DR ratio (R545/ R610) algorithm, using the intensity of the $\mathrm{HbO}_{2}$ absorption peak at $545 \mathrm{~nm}$ to that of the nonabsorbing wavelength of $610 \mathrm{~nm}$, for the detection of tissue pathologies using our non-invasive bimodal imaging system implemented on a multispectral imaging platform.

\subsection{Significance of Interquartile Range Values}

The spread of DR intensity ratios in clinically healthy, potentially malignant, and malignant lesions can be explained in terms of median and interquartile range (IQR) values. In the present study, clinically healthy tissues showed a median value of 1.41 for the R610/R545 image ratio and IQR of 0.55 (0.765 to 2.524), and no outliers were identified (range: 0.452 to 2.636).

For potentially malignant, the median was found to be 2.53 and the IQR was 0.98 (1.94 to 4.69). In the case of potentially malignant tissues, there are no outliers (1.055 to 4.985). For malignant tissues, the median was 3.589 and the IQR was 1.07 (2.132 to 8.189). About 5/49 of the malignant cases fall above the maximum range (2.29 to 5), indicating that these tissues may be more aggressive or have a poor prognosis. As compared with an earlier report, the median and IQR values in the present study are slightly higher but are within the desired range. ${ }^{19}$

\subsection{Site-Specific Algorithms and Effect on Diagnostic Accuracy}

Previous studies have demonstrated significant differences in the spectral properties between different anatomical sites in the oral cavity. ${ }^{37,38}$ Improvements in the tissue classification parameters such as PPV and NPV were observed when site-specific classification was used to discriminate head and neck SCC. ${ }^{39}$ To test this hypothesis on existing patient data, we collated data of patients with buccal mucosal abnormalities. We also recorded the DR ratio (R610/R545) from the left and right buccal mucosa of healthy volunteers with no history of alcohol, smoking, or tobacco usage. This data set was used to replace the patient data in the site-specific (buccal mucosa) scatterplot algorithm (Fig. 8) developed for discrimination of OPML and abnormal sites from patient normal data sets. The R610/R545 algorithm developed for buccal mucosa using healthy volunteer data instead of patient normal is shown in Fig. 9.

Table 2 shows the number of patients in each category, the discrimination line (DL) cut-off values, sensitivity, specificity, NPV, PPV, and the corresponding ROC-AUC values achieved for both cases. The improvement in the diagnostic accuracy observed on substitution of patient normal data with healthy volunteer buccal mucosa data was primarily an improvement in the specificity value from $96.3 \%$ to $100 \%$ with a concomitant increase in the PPV from 0.96 to 1 (Table 2), which was primarily due to the lowering of the DL from 2.214 to 2.112 . The corresponding ROCAUC curves for discrimination of OPML in the buccal mucosa of these patients are plotted in Fig. 10. The ROC-AUC values increased from 0.995 to 1 when healthy volunteer data were used instead of patient normal for detection of OPML sites in the buccal mucosa. 


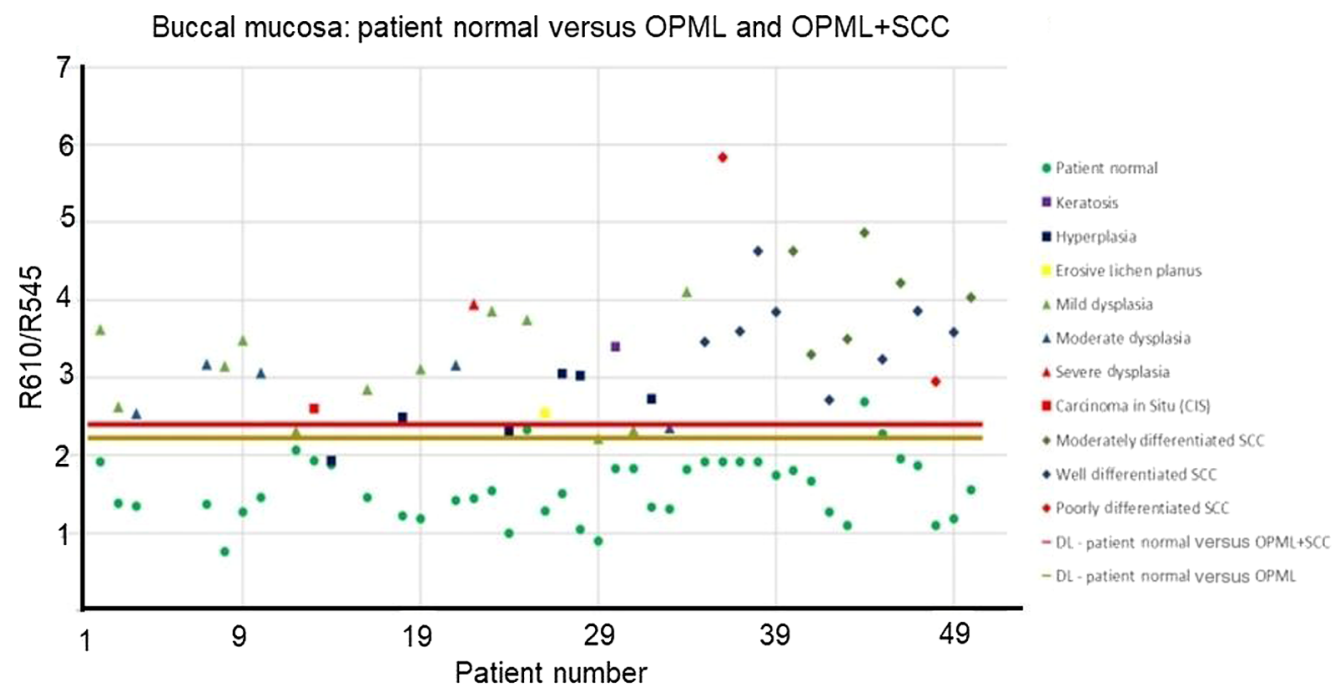

Fig. 8 Scatter plot algorithm of R610/R545 ratio for classifying OPML $(n=27)$ and abnormal (OPML + SCC) tissues $(n=43)$ of the buccal mucosa with patient normal. The red line discriminates abnormal tissues from patient normal, and the brown line discriminates OPML from patient normal.

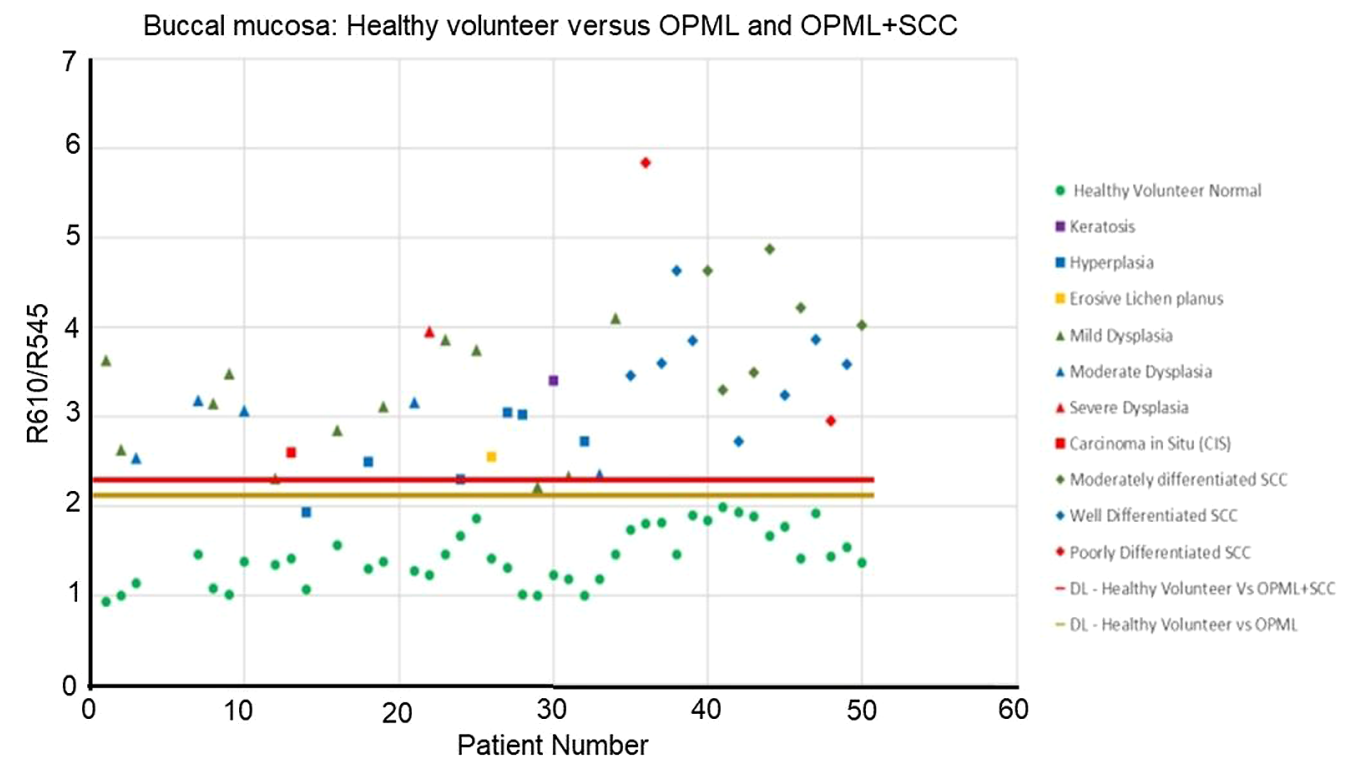

Fig. 9 Scatter plot algorithm of R610/R545 ratio for classifying OPML $(n=27)$ and abnormal (OPML + SCC) tissues $(n=43)$ of the buccal mucosa with healthy volunteer data from buccal mucosa. The red line discriminates abnormal tissues from healthy normal, and the brown line discriminates OPML from healthy normal.

\section{Discussions}

In recent times, there has been an increased interest in applying optical spectroscopy and imaging techniques for tissue diagnostics. These optical techniques are mostly non minimally or minimally invasive in nature, use non-ionizing radiation without any contrast agents, and have the ability to monitor patients over a period of time. The study results demonstrate the ability of using BMIS for screening and detection of OPMLs in the oral cavity. In addition, it can be used as a tool for guided biopsies by locating the most malignant site in the lesion for tissue biopsy and pathological confirmation of the grade of cancer with improved accuracy. This could potentially avoid multiple biopsies, minimize patient trauma, and reduce treatment costs. Furthermore, 
Subhash et al.: Bimodal multispectral imaging system with cloud-based machine learning algorithm...

Table 3 Showing the variation among intensity ratios using median and IQR.

\begin{tabular}{lcccc}
\hline \hline Tissue characteristics & Q1 & Q3 & Median & IQR (Min-Max) \\
\hline Healthy & 1.27 & 1.82 & 1.41 & $0.55(0.77-2.52)$ \\
Potentially malignant & 2.53 & 3.51 & 3.04 & $0.98(1.94-4.69)$ \\
Malignant & 3.11 & 4.18 & 3.59 & $1.07(2.13-8.19)$ \\
\hline \hline
\end{tabular}

the BIMS can be used to enhance the ability of the surgeon to locate the tumor margins, such that complete resection of the lesion is possible by providing enough margins during surgical interventions and minimizing functional deficits. The wavelength $(405 \mathrm{~nm})$ used in this study to estimate tissue abnormalities from autofluorescence matches with the Soret band of PpIX, which is a precursor in the heme production cycle. Since the ferrochelatase enzyme is inhibited in cancer cells, there is an increase in PpIX fluorescence from cancer cells. However, other biochemical constituents present in tissue that absorb at this wavelength, such as FAD and NADH, also absorb this light, and their concentration changes during cancer development could alter the overall emission characteristics of OPMLs.

The green LED light at $545 \mathrm{~nm}$ overlaps with the prominent absorption band of oxyhemoglobin located at $542 \mathrm{~nm}$. The red LED $(610 \mathrm{~nm})$ emission is at a non-absorbing wavelength for $\mathrm{HbO}_{2}$ and belongs to a region where scattering predominates and $\mathrm{Hb}$ has a higher absorption. Therefore, the diffusely reflected image of oral mucosa at $610 \mathrm{~nm}$ serves as a reference standard to map $\mathrm{HbO}_{2}$ absorption, as compared with using diffusely reflected light intensity at $575 \mathrm{~nm} \cdot{ }^{18,19,34}$ To confirm this, for the same set of data shown in Table 3, we plotted the scatterplot algorithm for the R545/R575 ratio and got a sensitivity of $37.5 \%$ and specificity of $86.2 \%$, with a PPV of 0.556 and NPV of 0.75 for classification of patient normal from OPML, with a cutoff values of 1.561 (Table S2 in the Supplementary Material). In comparison, the sensitivity and specificity obtained for the R610/R545 ratio algorithm were 97.5\% and 92.5\%, respectively, (Table 2) for discrimination of OPML from patient normal.

To test whether the high diagnostic accuracy for OPML is attributed to the large number of leukoplakia cases (14) involved in sample size (Table S1 in the Supplementary Material), we plotted the R610/R545 and R545/R575 ratio algorithms separately for leukoplakia cases involved in the study. It was seen that leukoplakia cases alone can be discriminated from normal with a sensitivity of $87.5 \%$ and specificity of $100 \%$ with the R610/R545 ratio algorithm as compared $97.5 \%$ and $87.5 \%$ for the algorithm covering all OPML cases with leukoplakia also included (Table S2 in the Supplementary Material).

Since tissue progression from normal tissues to OPML and SCC is accompanied by changes in tissue metabolism, structure, and morphology, these biochemical and morphological changes are manifested in the diffusely reflected spectral signatures derived from the tissue. ${ }^{40}$ We strongly believe that the wavelengths used in the present study, viz., 545 and $610 \mathrm{~nm}$, correlate to the changes in tissue vasculature and scattering during cancer development.

In the present study, apparently normal tissues of patients were taken as control. Also, the region contralateral to that of the diseased region or a few centimetres away from the OPML and free of inflammation was taken as control. The primary cause for malignancies in the head and neck region include smoking, alcohol consumption, and betel-quid chewing. ${ }^{41,42}$ The carcinogens associated with the development of malignancies in the head and neck region are known to not only induce alterations in the tumor site but also affect the entire organ site. ${ }^{43}$ This phenomenon is referred to as field cancerization (FC), and the changes manifested in the tissue due to FC include changes in tissue microvasculature, alterations in the nuclear size, and density. ${ }^{44,45}$ One of the issues attributed to the decreased diagnostic ability is the FC effects in the oral cavity of patients. To validate this, we compared the DR ratio (R610/R545) values from the buccal mucosa of healthy volunteers for the discrimination of potentially malignant lesions and noticed that the specificity values improved from $96.3 \%$ of $100 \%$ when healthy volunteer data were used instead of patient normal for buccal mucosal tissues (Fig. 9). The results are in agreement with our previous study, in which we implemented and established algorithms for all of the sites 
combined and an algorithm specifically for the buccal mucosa. ${ }^{19}$ The diagnostic ability of the algorithm specific to buccal mucosa was steadfastly higher for discriminating benign, dysplastic, and malignant lesions. In a similar study, Hu et al. ${ }^{39}$ reported an increase in PPV and NPV when using tissue-specific classification algorithms, which resulted in the false-positive rates declining by $34 \%$. The results from the present study indicate that site-specific algorithms implemented for the detection of lesions in the oral cavity increase diagnostic accuracy, which in turn reduces the number of false-negative rates. We also noticed that ulcers of the oral cavity show R610/R545 ratio values in the range of 2 to 2.6 (data not shown in Fig. 6), well below the malignant range (>3.6), which helps us to avoid unwanted biopsies from traumatic ulcers.

OSMF is characterized by oral inflammation, increase in submucosal collagen, and formation of fibrotic bands in the oral cavity that increasingly limit mouth opening. Increasing fibrosis causes blanching of oral mucosa, which results in a marble-like appearance. During the present study phase, nine OSMF cases were also screened, but they were excluded from the DR ratio algorithm and analysis as biopsies were not taken for histopathology. OSMF is a potentially malignant disorder with FC characteristics and biopsies usually taken only when clinical observations warrant it. The cases investigated had DR ratio (R610/R545) values ranging from 1.425 to 3.713 with a mean value of 2.51 , which corresponds to the premalignant range in our ML algorithm (Fig. 6).

Previously, various techniques based on optical spectroscopy and imaging have been evaluated to detect malignancies in the oral cavity. ${ }^{46-49}$ Cals et al. ${ }^{50}$ investigated the application of Raman spectroscopy for the intraoperative assessment of tumor margins in the prognosis of oral SCC. Linear discriminant analysis was used as a diagnostic algorithm to discriminate against healthy tissue types and oral SCC. One of the disadvantages of Raman spectroscopy is that the Raman effect is an extremely weak process in which 1 in $10^{6}$ to $10^{8}$ photons are Raman scattered. ${ }^{51}$ This weak phenomenon makes it extremely difficult to obtain a high-quality spectrum with the decreased integration times required for in vivo applications. Also, Raman spectroscopic approach requires high excitation power, extremely narrow bandwidths, and bulky and expensive instrumentation. ${ }^{52,53}$ Recently, a multimodal endomicroscopy incorporating hyperspectral and confocal imaging using a single foveated objective was developed and tested on ex vivo oral cancer samples. ${ }^{54}$ However, details regarding the diagnostic accuracy were not presented in the study. In the present study, we implemented a simple ratio-metric approach on the diffusely reflected light using the image intensity ratio R610/R545 that maps changes in oxygenated hemoglobin absorption in tissue for the detection of tumors in oral malignancies. The findings of the present study indicate a strong association between the increase in the red/green DR image ratio (R610/R545) and different types of oral precancers. With the use of site-specific algorithms and larger data sets, the diagnostic accuracy of the algorithms used for detection of OPMLs of the oral cavity would improve over time.

Furthermore, we believe that utilization of an ML algorithm provides a more reliable assessment of the cancer grade such that screening and diagnosis of cancer at the POC in real-time would become a reality. Additionally, the system incorporates fluorescence excitation at $405 \mathrm{~nm}$, which can be used as an additional tool for locating the tumor margins. Previous studies have established marked differences in the fluorescence emission profile with 400- to 410-nm excitation and were able to correctly identify 20 out of 22 samples investigated. ${ }^{55}$ The increase in the emission intensity at wavelengths greater than $600 \mathrm{~nm}$ noticed in this study can be credited to PpIX, which is accumulated in cancer cells owing to the reduced activity of the ferrochelatase enzyme in cancer cells. ${ }^{20}$ In yet another study, an optical imaging system with excitation at $405 \mathrm{~nm}$ was implemented for detection of precancerous lesions in the oral cavity. ${ }^{56}$ The changes associated with tissues during progression of neoplasia at this excitation wavelength include neovascularization in the stromal region and loss of auto-fluorescence due to the breakdown of collagen matrices. The use of optical fluorescence imaging or staining with toluidine blue may increase the number of lesions detected compared with oral visual examination alone and may increase border distinction at a subjective level. ${ }^{57}$ This technique will also be helpful in patients who are reluctant to have biopsy but are anxious to know the stage of their lesion. Overall, the results of this study indicate that the wide-field multimodal imaging incorporating fluorescence and DR will be of great significance in the screening of oral malignancies at the community level. 


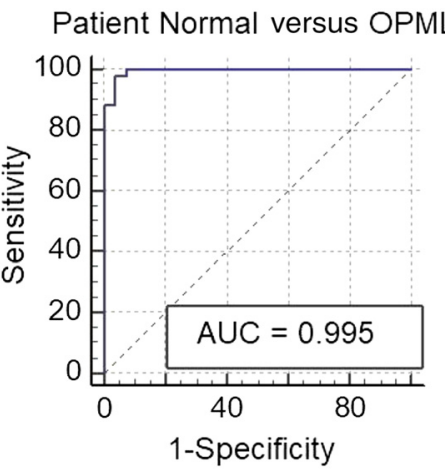

(a)

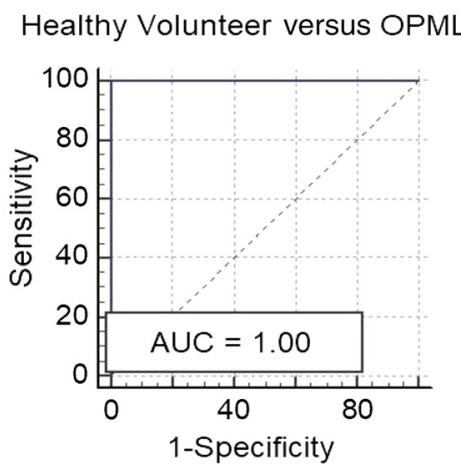

(b)

Fig. 10 ROC-AUC plots derived from the diagnostic accuracy values obtained from the R610/ R545 ratio algorithm for buccal mucosa, (a) patient healthy normal versus OPML sites and (b) healthy volunteer normal versus OPML.

\section{Conclusion}

The storage of data on a single cloud platform has multiple benefits. In addition to providing data safety, security, and ease of access to the screening centers, it facilitates compilation of screening data from different centers into an ML algorithm that provides real-time feedback to the device or the caregiver during the screening process. We believe that quantitative information on tissue status made available by the BMIS at the POC would lead to improved prognosis and patient management.

Different anatomical sites such as buccal mucosa, vermillion border of the lip, lateral and dorsal tongue, upper palate, and gingiva have different tissue structures, morphology, and reflectance characteristics. The light penetration and DR properties of various anatomical sites is different and could alter the sensitivity and specificity for detecting cancer at these sites. Therefore, site-specific algorithms, instead of one that incorporates data from all sites, will generate a higher diagnostic accuracy for screening of OPMLs. This has been validated in the case of patients under different stages of cancer in the buccal mucosa.

Although the BMIS was used to screen the oral cavity of around 350 people through this multicentric clinical trial, the clinical data presented relate to cases in which a biopsy was performed. In most cases in which a biopsy is not warranted or is ethically non-essential, the patients were advised to withdraw from their habits of pan or tobacco use and maintain oral hygiene. Since only two LEDs were used for each of the four wavelengths of light in the present design, uniformity of illumination was a major concern when the camera was to be used intraorally at short working distances from the tissue. Owing to this, the captured images were overexposed at some parts of the image, and the image data had to be discarded, despite repeated measurements. This was overcome by use of smaller form factor LEDs in a redesigned version of BMIS, currently being marketed by Sascan Meditech as OralScan ${ }^{\circledR}$. Based on user feedback, the GUI of proprietary software and its features were upgraded in this version of the device to provide an improved user experience. Since we are using an ML algorithm, more and more data are collated into the algorithm with the increasing number of users. We hope that in time we will be able to evolve site-specific algorithms and facilitate early detection of oral precancers with improved accuracy. We believe that only through mass screening and early detection of OMPLs of the oral cavity will it be possible to lower the high rates of mortality associated with the disease. The ability of the device to guide the clinician to the most malignant site in a lesion for tissue biopsy will minimize false negatives, unnecessary multiple biopsies, and unwanted treatments, leading to lower costs for disease management and reduced patient trauma.

\section{Disclosures}

The authors SN, SA, RP, SM, and RS are employees of Sascan Meditech, and SN, SM, and RS own stock in the company. SN also sits on Sascan's Board of Directors. However, the data 
analysis and reporting are free of bias. All other authors have no financial interest in this article and have no potential conflicts of interest to disclose.

\section{Acknowledgments}

The work presented in this paper was supported by grants from the Government of India under the Biotechnology Ignition Grant (BIG) scheme of Biotechnology Industry Research Assistance Council (BIRAC) and the INVENT and NIDHI-SSS schemes of the Department of Science \& Technology (DST), Govt of India, through Kalinga Institute of Information Technology Technology Business Incubator (KIIT-TBI), Bhubaneswar, and Technology Business Incubator for Medical Devices and Biomaterials (TIMed)-SCTIMST, respectively. The product development activities were also supported through the ELEVATE100 grant of Karnataka Biotechnology and Information Technology Services (KBITS), Government of Karnataka, and the productization grant of the Kerala Start-up Mission (KSUM), Government of Kerala. We sincerely acknowledge the cooperation of the patients who participated in the study and all support staff of the various organizations involved in the study, in particular, Dr Mukesh Kumar Das and Mr. Tanmaya Mohapatra of Reseapro Scientific Services, who coordinated the clinical studies in Bhubaneswar, Orissa. Thanks are also due to Dr Shyam Vasudev Rao of Renalyx Healthcare Pvt. Ltd. and to Dr. Ruhi Agarwala of UE-Life Sciences, for many fruitful discussions and comments.

\section{Code, Data, and Materials Availability}

The authors declare full availability of all data generated during the study and all material used for the analysis. Proprietary software was developed and used for operation of the device and data capture and analysis. No software code was associated with the main claims of the article. Table S1 in the Supplementary Material provides a list of all primary, processed, and pathological data used in the algorithm, and Table S2 in the Supplementary Material compares the diagnostic accuracies of the R610/R545 and R545/R575 ratios for discrimination of OPML and leukoplakia lesions from adjoining normal tissues of patients.

\section{Ethical Statements}

The multicenter clinical study followed the same protocols for patient enrollment, which was approved by the respective Ethical Committees of the Institutions involved, and the study was registered at the Clinical Trial Registry of India (CTRI). The Medial Devices and Diagnostic Division of the Central Drugs Standard Control Organization (CDSCO), Government of India, has certified that the device OralScan ${ }^{\circledR}$ is not notified under Section 3(b)(iv) of the Drugs and Cosmetics Act 1940.

\section{References}

1. F. Bray et al., "Global cancer statistics: GLOBOCAN estimates of incidence and mortality worldwide for 36 cancers in 185 countries," CA: A Cancer J. Clin. 68, 394-424 (2018).

2. M. P. Singh et al., "Clinical profile and epidemiological factors of oral cancer patients from North India," Natl. J. Maxillofac. Surg. 6, 21-24 (2015).

3. S. B. Thavarool et al., "Improved survival among oral cancer patients: findings from a retrospective study at a tertiary care cancer centre in rural Kerala, India," World J. Surg. Oncol. 17(1), 15 (2019).

4. S. S. Napier and P. M. Speight, "Natural history of potentially malignant oral lesions and conditions: an overview of the literature," J. Oral Pathol. Med. 37, 1-10 (2007).

5. H. Mortazavi, M. Baharvand, and M. Mehdipour, "Oral potentially malignant disorders: an overview of more than 20 entities," J. Dent. Res. Dent. Clin. Dent. Prospect. 8(1), 6-14 (2014). 
6. M. W. Lingen et al., "Critical evaluation of diagnostic aids for the detection of oral cancer," Oral. Oncol. 44, 10-22 (2008).

7. N. L. Rhodus, A. R. Kerr, and K. Patel, "Oral cancer. Leukoplakia, premalignancy, and squamous cell carcinoma," Dent. Clin. N. Am. 58, 315-340 (2014).

8. A. Panwar, R. Lindau, and A. Wieland, "Management for premalignant lesions of the oral cavity," Expert Rev. Anticancer Ther. 14, 349-357 (2014).

9. M. D. Mignogna, S. Fedele, and L. Lo Russo, "Dysplasia/neoplasia surveillance in oral lichen planus patients: a description of clinical criteria adopted at a single centre and their impact on prognosis," Oral Oncol. 42, 819-824 (2006).

10. J. B. Epstein et al., "Analysis of oral lesion biopsies identified and evaluated by visual examination, chemiluminescence and toluidine blue," Oral Oncol. 44(6), 538-544 (2008).

11. M. W. Lingen et al., "Evidence-based clinical practice guideline for the evaluation of potentially malignant disorders in the oral cavity: a report of the American Dental Association," J. Am. Dent. Assoc. 148, 712-727.e10 (2017).

12. X. M. Ang et al., "Quality of life in patients with oral leukoplakia," J. Oral Pathol. Med. 48, 574-580 (2019).

13. R. Macey et al., "Diagnostic tests for oral cancer and potentially malignant disorders in patients presenting with clinically evident lesions," Cochrane Database Syst. Rev. 2015, CD010276 (2015).

14. E. C. Yang et al, "Development of an integrated multimodal optical imaging system with real-time image analysis for the evaluation of oral premalignant lesions," J. Biomed. Opt. 24, 025003 (2019).

15. S. Fedele, "Diagnostic aids in the screening of oral cancer," Head Neck Oncol. 1, 5 (2019).

16. E. Duran-Sierra, "Clinical label-free biochemical and metabolic fluorescence lifetime endoscopic imaging of precancerous and cancerous oral lesions," J. Oral Oncol. 105, 104635 (2020).

17. D. Yong et al., "Autofluorescence spectroscopy in redox monitoring across cell confluencies," PLOS One 14(12), e0226757 (2019).

18. N. Subhash et al., "Oral cancer detection using diffuse reflectance spectral ratio R540/R575 of oxygenated hemoglobin bands," J. Biomed. Opt. 11(1), 014018 (2006).

19. R. J. Mallia et al., "Oxygenated hemoglobin diffuse reflectance ratio for in vivo detection of oral precancer," J. Biomed. Opt. 13(4), 041306 (2008).

20. R. J. Mallia et al., "Laser-induced autofluorescence spectral ratio reference standard for early discrimination of oral cancer," Cancer 112(7), 1503-1512 (2008).

21. M. M. Stephen et al., "Diagnostic accuracy of diffuse reflectance imaging for early detection of pre-malignant and malignant changes in the oral cavity: a feasibility study," BMC Cancer 13, 278 (2013).

22. S. P. Singh et al, "Recent advances in optical diagnosis of oral cancers: review and future perspectives," Head Neck 38, E2403-E2411 (2016).

23. T. M. Bydlon et al., "Chromophore based analyses of steady-state diffuse reflectance spectroscopy: current status and perspectives for clinical adoption," J. Biophotonics 8(1-2), 9-24 (2015).

24. D. C. G. de Veld et al., "Autofluorescence and diffuse reflectance spectroscopy for oral oncology," Lasers Surg. Med. 36, 356-364 (2005).

25. R. A. Schwarz et al., "Autofluorescence and diffuse reflectance spectroscopy of oral epithelial tissue using a depth-sensitive fiber-optic probe," Appl. Opt. 47, 825-834 (2008).

26. M. J. Bailey et al., "Detection of precancerous lesions in the oral cavity using oblique polarized reflectance spectroscopy: a clinical feasibility study," J. Biomed. Opt. 22(6), 065002 (2017).

27. D.V. Messadi, "Diagnostic aids for detection of oral precancerous conditions," Int. J. Oral Sci. 5, 59-65 (2013).

28. R. S. Ganga et al., "Evaluation of the diagnostic efficacy and spectrum of autofluorescence of benign, dysplastic and malignant lesions of the oral cavity using VELscope," Oral Oncol. 75, 67-74 (2017).

29. J. Fourie, "VELscope: shedding light on its ideal application," S. Afr. Dent J. 73(2), 71-77 (2018). 
30. M. Mascitti et al., "An overview on current non-invasive diagnostic devices in oral oncology," Front. Physiol. 9, 1510 (2018).

31. D. V. Messadi et al., "The clinical effectiveness of reflectance optical spectroscopy for the in vivo diagnosis of oral lesions," Int. J. Oral. Sci. 6(3), 162-167 (2014).

32. Y. Lalla et al., "Assessment of oral mucosal lesions with autofluorescence imaging and reflectance spectroscopy," J. Am. Dent. Assoc. 147(8), 650-660 (2016).

33. N. Subhash, "Hand-held biophotonic medical device, method, and system for multimodal and multispectral imaging of tissue," Indian Patent \# 303949 (2018); USPTO Pub No. 2020/0196869 A1 (2020).

34. Y. Guan and V. Wong, "Periodontal disease detection system and method," US Patent No. US 10,039,489B2 (2018).

35. K. Soklov et al., "Reflectance spectroscopy with polarized light: is it sensitive to cellular and nuclear morphology," Opt. Express 5, 302-317 (1999).

36. C. S. Prasanth et al., "Discrimination of periodontal diseases using diffuse reflectance spectral intensity ratios," J. Biomed. Opt. 17(2), 027001 (2012).

37. D. C. G. de Veld et al., "Autofluorescence characteristics of healthy oral mucosa at different anatomical sites," Lasers Surg. Med. 32(5), 367-376 (2003).

38. A. Gillenwater et al., "Noninvasive diagnosis of oral neoplasia based on fluorescence spectroscopy and native tissue autofluorescence," Arch. Otolaryngol. Head Neck Surg. 124(11), 1251-1258 (1998).

39. F. Hu et al., "Assessment of the sensitivity and specificity of tissue-specific-based and anatomical-based optical biomarkers for rapid detection of human head and neck squamous cell carcinoma," Oral Oncol. 50(9), 848-856 (2014).

40. K. Sokolov et al., "Optical spectroscopy for detection of neoplasia," Curr. Opin. Chem. Biol. 6(5), 651-658 (2002).

41. N. Guha et al. "Betel quid chewing and the risk of oral and oropharyngeal cancers: a meta-analysis with implications for cancer control," Int. J. Cancer 135(6), 1433-1443 (2014).

42. S. Petti, M. Masood, and C. Scully, "The magnitude of tobacco smoking-betel quid chewing-alcohol drinking interaction effect on oral cancer in South-East Asia. A meta-analysis of observational studies," PLoS One 8(11), e78999 (2013).

43. L. Kopelovich et al., "Surrogate anatomic/functional sites for evaluating cancer risk: an extension of the field effect," Clin. Cancer Res. 5(12), 3899-3905 (1999).

44. O. Bugter et al, "Optical detection of field cancerization in the buccal mucosa of patients with esophageal cancer," Clin. Transl. Gastroenterol. 9(4), 152 (2018).

45. H. Subramanian et al., "Nanoscale cellular changes in field carcinogenesis detected by partial wave spectroscopy," Cancer Res. 69(13), 5357-5363 (2009).

46. M.-J. Jeng et al., "Raman spectroscopy analysis for optical diagnosis of oral cancer detection," J. Clin. Med. 8(9), 1313 (2019).

47. J. Vonk et al., "Improving oral cavity cancer diagnosis and treatment with fluorescence molecular imaging," Oral Diseases 27(5), 21-26 (2020).

48. S. P. Sunny et al., "Intra-operative point-of-procedure delineation of oral cancer margins using optical coherence tomography," Oral Oncol. 92, 12-19 (2019).

49. P. Panta et al., "Optical coherence tomography: emerging in vivo optical biopsy technique for oral cancers," in Oral Cancer Detection, P. Panta, Ed., pp. 217-237, Springer International Publishing (2019).

50. F. L. J. Cals et al., "Investigation of the potential of Raman spectroscopy for oral cancer detection in surgical margins," Lab. Invest. 95(10), 1186-1196 (2015).

51. R. R. Jones et al, "Raman techniques: fundamentals and frontiers," Nanoscale Res. Lett. 14(1), 231 (2019).

52. C. Eliana et al., "In-vivo Raman spectroscopy: from basics to applications," J. Biomed. Opt. 23(7), 071210 (2018).

53. H. Chen et al., "Identification and characterization of bladder cancer by low-resolution fiberoptic Raman spectroscopy," J. Biophotonics 11(9), e201800016 (2018).

54. A. Shadfan et al., "Development of a multimodal foveated endomicroscope for the detection of oral cancer," Biomed. Opt. Express 8(3), 1525-1535 (2017). 
55. D. R. Ingrams et al., "Autofluorescence characteristics of oral mucosa," Head Neck 19(1), 27-32 (1997).

56. P. Lane et al., "Oral fluorescence imaging using 405-nm excitation, aiding the discrimination of cancers and precancers by identifying changes in collagen and elastic breakdown and neovascularization in the underlying stroma," Gend. Med. 9, S78-S82 (2012)

57. S. Warnakulasuriya, "Oral potentially malignant disorders: a comprehensive review on clinical aspects and management," Oral Oncol. 102, 104550 (2020).

Subhash Narayanan is the founder CEO and CTO of Sascan Meditech. He received his $\mathrm{PhD}$ in lasers from Cochin University of Science and Technology (CUSAT), Kochi, and has been working in the domain of biophotonics research for the past two decades at the National Centre for Earth Science Studies (NCESS), Thiruvananthapuram. His research interests include point monitoring and multispectral imaging of oral and cervical tissues, remote sensing of vegetation and corals, and application of photodynamic therapy in the management of early-stage cancers and gingival/periodontal inflammation. He was Marie Curie Fellow at the Institute of Quantum Electronics-CNR, Florence, Italy, and a senior DAAD Fellow at the Botanical Institute, University of Karlsruhe, Germany. He was conferred with the Novartis Oration Award in 2010 by the Indian Council of Medical Research (ICMR) for his contributions in cancer research.

Suresh Anand received a BEng degree in electronics and instrumentation from Bharathidasan University, India, master's degree in laser and electro-optical engineering from the College of Engineering Guindy, Anna University, India, and his $\mathrm{PhD}$ in biomedical engineering from the Indian Institute of Technology Madras, India. During his $\mathrm{PhD}$, he focused on the application of quantitative diffuse reflectance spectroscopy for monitoring the wound healing process in diabetic foot ulcers. He was a research fellow in the National Institute of Optics-National Research Council (INO-CNR) and European Laboratory for Non-Linear Spectroscopy (LENS), Florence, Italy. His research interests include the application of tissue optical point-spectroscopy with a primary focus towards tumor detection and especially interested in first-in-human clinical studies.

Ranimol Prasanna received her master's degree in dental surgery from Government Dental College, Trivandrum, with specialization in oral medicine and radiology. She is committed in oral cancer diagnosis and treatment of potentially malignant oral disorders as well as dental hard and soft tissue disorders. She is interested in molecular diagnostics underlying the process of carcinogenesis. Currently, she is pursuing her $\mathrm{PhD}$ at the University of Kerala in the application of salivary biomarkers for early detection of oral cancer by monitoring changes in cytokine levels.

Sandeep P. Managoli received his graduation in electronics and communication engineering from the RNS Institute of Technology, Bengaluru. He is an experienced software developer with expertise in the latest software tools. Before joining Sascan, he was with the Philips Healthcare, Bengaluru, as software engineer. He has a demonstrated history of working with customeroriented solutions and is presently engaged in developing software solutions for various products of the company.

Rinoy Suvarnadas has a BTech degree in mechanical engineering from CUSAT, Kochi. He also holds a diploma in digital manufacturing from Fab Academy, USA. He is a certified ISO 13485:2016 Internal Auditor. As senior manager, he is involved in the implementation of quality management systems at Sascan. He is interested in 3D designing, prototyping and is involved in $\mathrm{R} \& \mathrm{D}$ activities and product development.

Vidyarani Shyamsundar is professor and principal investigator in the Centre for Oral Cancer Prevention Awareness and Research at Sree Balaji Dental College and Hospital, Chennai. She completed her MDS in oral pathology and microbiology from the Government Dental College, Trivandrum, Kerala. Her research focuses on the clinicopathological and molecular aspects of oral squamous cell carcinoma and oral potentially malignant disorders. 
Karthika Nagarajan is an associate professor in the Department of Oral Pathology and Microbiology, Sree Balaji Dental College, Chennai. She is also associated as a researcher at the Centre for Oral Cancer Prevention, Awareness \& Research (COCPAR), leading to her PhD in oral pathology.

Sourav K. Mishra is a medical oncologist currently practicing at IMS \& SUM Hospital, Bhubaneswar. His fields of interest include hematological malignancies, breast and thoracic oncology, gynecological oncology, gastrointestinal oncology, and genitourinary oncology. He is a recipient of the Best Medical Oncologist 2021 Award and the Times Health Icon Excellence Award. He along with his team strives to provide the best comprehensive care to cancer patients.

Migi Johnson is a post-graduate student in the Department of Oral Medicine and Radiology at the Government Dental College, Kottayam, Kerala. Her mission is to provide comprehensive dental services to all patients who need care. Her research focuses on improving specialized training and experience in the diagnosis and management of oral mucosal abnormalities, including oral cancer, potentially malignant disorders, and orofacial pain.

Mahesh Dathurao Ramanand is a professor in oral medicine and radiology with two decades of experience in oral and maxillofacial surgery. He received his BDS from V.S. Dental College, Bengaluru, and MDS from Bangalore Institute of Dental Sciences Hospital, Bengaluru. His areas of interest are periodontal care, dental implant fixing, geriatric dental care, temporo-mandibular disorders, and orthodontics.

Sanjay C Jogigowda is an oral physician and maxillofacial radiologist with more than a decade of clinical and research experience. His clinical interests are in the diagnosis and management of oro-mucosal diseases, oral potentially malignant disorders, and orofacial pain. He loves embracing encounters and derives satisfaction by providing high-quality oral health care for patients with complex medical conditions and diagnostic challenges.

Vishal Rao is presently the chief of Head \& Neck Surgical Oncology \& Robotic surgery at HCG Cancer Centre, Bengaluru. His areas of expertise include surgery for benign and malignant tumors of the thyroid, parotid, and salivary gland surgeries, complex neck surgeries, such as radical and modified neck dissections, carotid body, other vascular tumors, and parapharyngeal space tumors. He is the inventor of "Aum Voice Prosthesis," which is a 1-dollar speaking device for throat cancer patients. In recognition to his contributions, he has been conferred the Honorary FRCS from Royal College of Surgeons in Glasgow. He has also received Judy Wilkenfeld Award for Global Excellence in tobacco control. He is an international advisor to World Health Organization Tobacco Free initiatives and is a visiting faculty of John Hopkins University leadership programs.

Kodaganur S. Gopinath is a surgical oncologist in the field of head and neck cancer. He has specialized in head and neck services at the Memorial Sloan Kettering Cancer Center, New York, as a visiting fellow, and MD Anderson Cancer Center, Houston, Texas. He received his Hon. FRCS from RCS Edinburgh, UK. His main interests lie in breast cancer, head and neck cancer, and oral and cervical cancer surgery. He is the recipient of the prestigious Padmashri Award for his excellent work in the field of cancer treatment from the Hon. President of India. He also received the Dr. BC Roy National Award of the Indian Council of Medical Research (ICMR). 